\title{
Neue Gewerkschaft für die Pflege
}

\section{Unabhängig zu besserer Interessenvertretung für optimale Arbeitsbedingungen}

Die Unzufriedenheit mit der gewerkschaftlichen Vertretung in Sachen Arbeitszeit, Arbeitsverdichtung und Bezahlung führte nun im Oktober zur Gründung einer neuen Gewerkschaft Pflege. Nach dem Vorbild der Asklepios Gewerkschaft für angestellte Ärztinnen und Ärzte ist die Pflegegewerkschaft unabhängig und sieht sich damit besser in der Lage, die Interessen ihrer Berufsgruppe zu vertreten. Ziel ist ein Dachverband der unabhängigen Gewerkschaften der Gesundheitsberufe Österreich. Das personelle Potential wäre damit beträchtlich, wieweit sich dieses tatsächlich mobilisieren lässt, bleibt abzuwarten.

Auslöser für die Gründung der neuen Pflegegewerkschaft war das Krankenanstalten-Arbeitszeitgesetz, das nicht nur, wie in der Öffentlichkeit überwiegend vermittelt, die Ärzte betrifft. Während diese jedoch mit traditionell wirkungsvoller Einflussnahme deutliche Verbesserungen ihrer Rahmenbedingungen erreichen konnten, sehen die Initiatoren und Gründungsmitglieder der Gewerkschaft Pflege, Fabian Martin und Lukas Ertler, die Gefahr, dass die Pflege in dieser Situation zum Assistenzberuf für Ärzte umfunktioniert werden könnte. „Die Kernkompetenz der Pflege ist die eigenverantwortliche Arbeit am Patienten", konstatierte Martin bei der Präsentation der neuen Berufsvertretung. Die extreme Arbeitsverdichtung in der Folge der Arbeitszeitverkürzung für Ärzte führe dagegen zu Konflikten zwischen den Berufsgruppen. Womit auch eines der Hauptthemen angesprochen werden soll: Partnerschaftliche Beziehungen aller Gesundheitsberufe. Diese sollen auch durch den Informations- und Erfahrungsaustausch mit der Asklepios Gewerkschaft gepflegt, und durch den geplanten Dachverband gestärkt werden.

\section{Zuständigkeiten} im ÖGB zersplittert

Im Österreichischen Gewerkschaftsbund (ÖGB) wird die Pflege derzeit von verschiedenen Gewerkschaften präsentiert, je nachdem, ob es sich um Privat- oder öf-

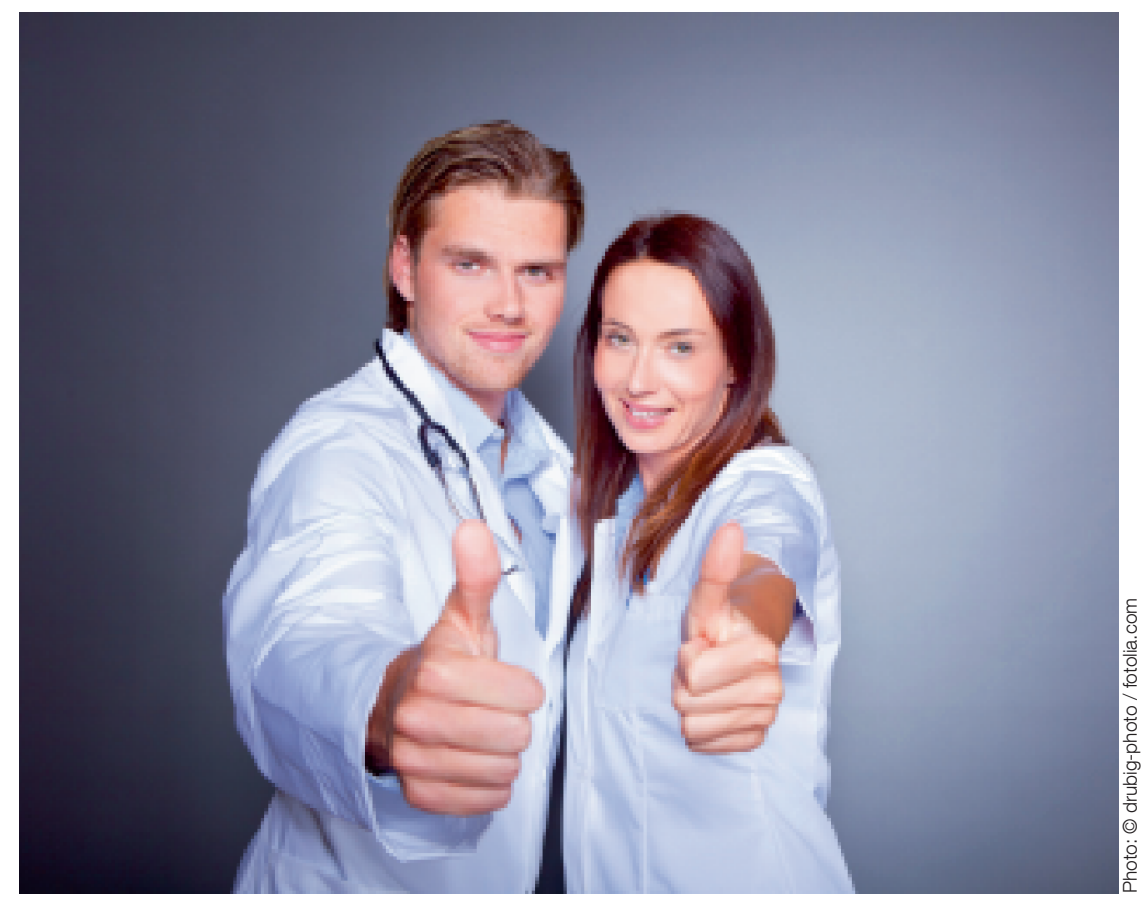

Pflege, Arzt und andere Gesundheitsmitarbeiter als Team.

fentliche Krankenanstalten handelt, in welchen die Pflegekraft tätig ist. Hier sollten die Rahmenbedingungen für die Pflege ausverhandelt werden. Ein hoher Anteil von Pflegepersonen, die an psychischer und körperlicher Erschöpfung leiden, und eine hohe Fluktuation in Pflegeberufen deuten für Martin und Ertler darauf hin, dass diese Rahmenbedingungen nicht optimal sind. Während die Gründung einer Gewerkschaft in Österreich ein Grundrecht ist, verfügen nur die Gewerkschaften, die im ÖGB organisiert sind, über „Kollektivvertragsfähigkeit“, werden also von den Sozialpartnern als Verhandlungspartner anerkannt. Einstweilen muss die Gewerkschaft Pflege sich also auf Bewusstseinsbildung bei Politik und Öffentlichkeit - und wohl auch bei der eigenen Berufsgruppe - beschränken.

Zum Österreichischen Krankenpflegeverband (ÖGKV), der sich in den vergangenen Jahren in ganz konkreten Sachfragen als Verhandlungspartner etablieren konnte und mit jahrelanger Hartnäckigkeit schließlich zum Beschluss der Reform des Gesundheits- und Krankenpflegege- setzes und des Berufsregistergesetzes im vergangenen Sommer wesentlich beigetragen hat, sieht sich die neue Gewerkschaft nicht als Konkurrenz. Die Zielrichtung der Gewerkschaft sei die Verhandlung der Kollektivverträge, so Martin. Man möchte durchaus das Gespräch mit dem ÖGKV suchen. Für Ursula Frohner, Präsidentin des ÖGKV, zeigt die Neugründung deutlich das Vakuum, das nicht zuletzt durch den besonders hohen Reformdruck auftrete. „Wir sind interessiert an der Zielsetzung und Umsetzung“, stellt Frohner fest. Wichtig wäre allerdings, die Kräfte der Pflege, der mit 65 Prozent der Mitarbeiter größten Berufsgruppe im Gesundheitsbereich, zu bündeln.

Informationen: www.pflege-gewerkschaft.at Quelle: Pressekonferenz Pflege Gewerkschaft und Asklepios Gewerkschaft, 26. 9. 2016, Wien

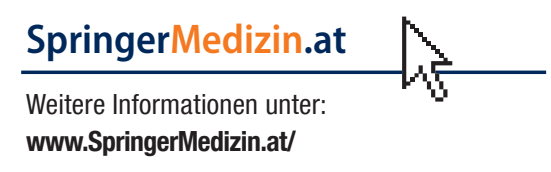

